\title{
The Status Quo of Inheritance Education of Famous Experts in Traditional Chinese Medicine in China
}

\author{
Junping Zhang ${ }^{1}$, Aihua Zhang ${ }^{2}$, Zheng Wei ${ }^{3}$ and Yi Zhao ${ }^{4}$ \\ Oncology Department,Henan Academy institute of Traditional Chinese Medicine, Zhengzhou, Henan Province \\ 450000,china \\ ${ }^{1}$ zhjunpingi0372@163.com, ${ }^{2} 18037128591 @ q q . c o m$, \\ ${ }^{3}$ questwz@163.com , ${ }^{4} 277789196 @ q q . c o m$ \\ *Corresponding author: 277789196@qq.com
}

\begin{abstract}
Traditional Chinese Medicine (TCM) is a very practical subject; and clinical experience is its cornerstone. The biggest difference between TCM and Western medicine lies in the particularity of its inheritance. Chinese medicine is an empirical medicine. This paper mainly discusses how to better inherit traditional Chinese medicine through the mode of famous experts in traditional Chinese medicine teaching their apprentices. 2. Methods: By studying the unique syndrome differentiation methods of famous experts in traditional Chinese medicine, the unique therapeutic principles and methods, unique medical technology and the unique inheritance method of effective prescriptions, a national service platform of acquisition and inheritance of academic experience of famous experts in Chinese traditional Chinese Medicine is researched and developed, the methods of inheritance and application will be further studied, so as to further promote the inheritance effect and promote the academic and clinical development of TCM. 3. Results: State Administration of Traditional Chinese Medicine of the People's Republic of China has set up two batches of 433 Chinese Studios, which inherit and develop the academic thoughts and clinical experience of tutors teaching their apprentices. And a long-term mechanism of research and inheritance is established, promoting the clinical and academic progress of Chinese Medicine. 4. Conclusions: Through the establishment of the studios of famous experts in traditional Chinese medicine, a large number of research results have been achieved, and a large number of outstanding Chinese clinical talents have been cultivated, which drives the clinical development of Chinese medicine, and promotes the inheritance and innovation of Chinese medicine theory, and has a good social impact.
\end{abstract}

Keywords-Traditional Chinese Medicine; Inheritance Education; Famous Experts; Theory Innovation; China

\section{INTRODUCTION}

Traditional Chinese medicine is the product gradually formed in the process of struggling with the disease in the long term for the Chinese nation by combining theory and practice, is the understanding of health and disease summarized by the ancient physicians and different academic schools through continuous in-depth observation and repeated clinical practice ${ }^{[1]}$. It is rooted in the fertile soil of Chinese traditional culture, is a double-attribute subject of natural science and humanities led by the philosophy. To study and use the wisdom of the Chinese nation, to inherit and carry forward the academic essence of Chinese medicine is an important mission to develop a new generation of Chinese medicine talents. Chinese medicine theory comes of the summary of medical experience and ancient Chinese Yin-yang and Five-element thoughts, the traditional Chinese medicine theory system is gradually formed under the guidance of materialism and dialectical thoughts through the long-term clinical practice, it comes from practice, in turn, and it guides the practice ${ }^{[2]}$. The unique theoretical system of traditional Chinese medicine has two basic characteristics, one is the overall concept, and the other is the syndrome differentiation. The basic theory of traditional Chinese medicine is a theoretical summary of the changes of human life activities and diseases. Inheritance is the main form of the continuation of Chinese academic experience. As a representative of the highest level of academic experience, the famous experts in traditional Chinese medicine have been an important carrier and object of the research of traditional Chinese medicine. In the previous study of inheritance, people have carried out many useful explorations, and they summarized the inheritance modes as the following basic forms: the mentoring-type inheritance, college-type inheritance, and research-type inheritance; The research methods are summarized as the following basic methods: the organization research based on modern information technology, inheritance research based on the clinical application, inheritance based on the 
innovation theory.

\section{THE NECESSITY OF INHERITING THE FAMOUS}

\section{EXPERTS IN TRADITIONAL CHINESE MEDICINE}

The famous experts in traditional Chinese medicine are the group with the most profound TCM academic attainments and the highest clinical level. They are the typical example of the combination of TCM theory, previous experience and present clinical practice, and an important carrier of the knowledge of Chinese medicine ${ }^{[3]}$. The wise and able persons and famous doctors through the ages are familiar with the classic cases, are diligent in the clinical cases, they can bring forth the new through the old, and create a new theory. Their experience is a combination of profound traditional Chinese medicine theory and rich clinical experience; their continuous inheritance and innovation of traditional Chinese medicine has promoted the enrichment and development of TCM theory system. Compared with the vast ancient literature of traditional Chinese medicine, it is more vivid and lively, and is of more practical guidance. It is because of the accumulation and inheritance of the experience of famous experts in traditional Chinese medicine, which makes traditional Chinese medicine eternally renewed, keep the vitality. Research and inheritance of the famous experts' clinical experience and academic thought in traditional Chinese medicine is an important part of the development of traditional Chinese medicine theory and practice, the "mentoring-type inheritance" of traditional Chinese medicine is the traditional Chinese way of inheritance, and is an important way to inherit the legacy of the famous experts in traditional Chinese medicine, develop TCM cause and cultivate the talents. The "mentoring-type inheritance" makes the famous experts' valuable experience accumulated in the long-term clinical practice pass down from generation to generation. In recent years, Chinese medicine management departments at all levels attaches great importance to the implementation and popularization of TCM apprentice education projects, especially, they have achieved great improvement in the aspect of the basic traditional Chinese medicine services.

\section{CONSTRUCTION OF FAMOUS EXPERTS' STUDIOS IN}

\section{TRADITION CHINESE MEDICINE}

In 2012, the State Administration of Traditional Chinese Medicine of the People's Republic of China set up 210 national studio construction projects for the famous experts in traditional Chinese medicine, and in 2014, it set up 223 national studio construction projects for the famous experts in traditional Chinese medicine, too; 433 people in total in 2 batches ${ }^{[4]}$. The key work of the studios of the famous experts in traditional Chinese medicine is the inheritance and exploration of the mentoring-type inheritance academic thoughts and clinical experience in the studio, to show a distinct personality characteristic; which is different from the popular, basic education of colleges and universities. The studio of traditional Chinese medicine is a deeper level of education, through the ways of "one to one", or "one to many", it has a relatively smaller size than that of the training course, but the inheritance of the mentor's experience is more in-depth, so it is needed to extract unique experience in medicine and academic ideas from clinical and traditional Chinese medicine.

\section{A. The summary of the academic thoughts of the schools}

The academic attainments of the famous experts in traditional Chinese medicine are great and their skills are superb, they are the typical example of the combination of TCM theory, previous experience and present clinical practice. The inheritance of their academic thoughts and clinical thinking is an important job of the studio of the famous experts in traditional Chinese medicine. So it is needed to summarize the academic thought and dialectical thinking of the famous experts in TCM, and publish related papers and books, make them widely popular. Taking the effective prescription of the famous experts in traditional Chinese medicine as the research object, through retrospective case analysis, prospective system case study, the paper further standardizes and evaluates the effective prescription, forming the clinical application guidance for effective prescription, and the specification of effective prescription and evaluation and inheritance of the effective prescription. Through the guidance of the famous experts in TCM, the studios can create the atmosphere of the traditional culture, deepen the study of the background knowledge, understand the carrier of traditional Chinese culture, and on this basis, the studio should intensively read the classics. There is no short cut in the study of TCM, we must be down-to-earth, pay attention to basic skills, attach importance to the understanding of classics; although most famous doctors are in senior age, they can read the classics. The classics of traditional Chinese medicine combined the wisdom crystallization of the wisdom of the ancients and clinical experience; the experience of the famous experts in traditional Chinese medicine can lead us to a highly effective grasp of the knowledge of traditional Chinese medicine ${ }^{[5]}$.

\section{B. Inheritance of traditional Chinese medicine clinical}

\section{diagnosis and treatment technology}

Being engaged in traditional Chinese medicine diagnosis and treatment for decades, the famous experts in traditional Chinese medicine have accumulated a lot of valuable experience. The clinical diagnosis and treatment technology and experience cannot be learned from books, only be studied with the help of the teachers. Only after summarizing the dialectical thought and drug laws of the diagnosis and treatment in oral face-to-face, can the students obtain get real skills of clinical experience from the famous experts in traditional Chinese medicine. The establishment of TCM studio for the famous experts, organizing the teacher notes, teacher medical cases, then, by publishing textbooks, books, a special disease treatment, medical records, these can be more conveniently inherited and researched. Such as Shi Xuemin's acupuncture treatment for hypertension, He Puren's three acupuncture technique in treatment of stroke. Through standard and evaluation research, the technical operation specification, indications, and clinical effects of the traditional Chinese medicine characteristic diagnosis and treatment technology are further clarified, so as to 
develop the clinical diagnosis and treatment technology scheme and audio-visual materials for popularization and application, and then to explore the diagnosis and treatment program ${ }^{[6]}$. The unique and effective prescription refers to unique and effective clinical prescription of the famous experts applied in traditional Chinese medicine. Through a retrospective case analysis of data, prospective system case study, and effective prescriptions is further standardized and evaluated, then effective prescriptions clinical application guidelines are formed for the popularization and application and the specifications, evaluation and appropriate method of effective prescriptions.

\section{Inheritance of research results}

The academic experience content of the famous experts in traditional Chinese medicine is profound and flexible, which is not conducive to the study of learners, and it limits its popularization and application as well. The academic experience of famous experts in traditional Chinese medicine is transformed into scientific research results, which is beneficial to its inheritance and development, and is also conducive to the promotion and application. Inheritance of the scientific research achievements is also one of the jobs of the studio of the famous experts in traditional Chinese medicine, and an innovative way as well, which is conducive to the improvement of clinical efficacy. Encouraging the heirs to boldly use new technologies, new methods for active exploration on traditional Chinese medicine treatment of prescription drugs, and a certain results have been achieved, and SPSS statistical software is applied for data $\operatorname{mining}{ }^{[7]}$.

\section{Cultivation of high-quality TCM talents}

The construction of the studio of the famous experts in traditional Chinese medicine should not only inherit and develop the academic thoughts of the famous experts in traditional Chinese medicine, but also cultivate high-quality talents for the society, so that more patients can benefit from the diagnosis and treatment of traditional Chinese medicine. Traditional TCM inheritance modes are mentoring-type inheritance and the family-type inheritance. Since the founding of the PRC, the strong development of colleges and universities in TCM makes the proportion of education in Colleges and universities increase, and college-type education has become an important way to cultivate talents ${ }^{[8]}$. This kind of training method is unified, the teachers teach knowledge, the students lack the practice, which cannot fully develop the ability of each student for TCM syndrome differentiation, but the college education can cultivate the students majoring in traditional Chinese medicine how to use the modern medical equipment. The construction of studios for the famous experts in traditional Chinese medicine should be based on the convenience of the University of Traditional Chinese Medicine, widely train talents of traditional Chinese medicine, and to focus on the cultivation of inheritors for the schools. In recent years, the cultivation quality of talents of the traditional Chinese medicine, the problems existing in TCM education have caused wide attention from the national authorities and the education sector of the Chinese medicine, and these problems are taken as a major project for demonstration research; the landslide phenomenon of the traditional Chinese medicine teaching has begun to be recognized, and more and more people realize that the important position of TCM in TCM education should not be shaken, the classical theory is the foundation of traditional Chinese medicine, and it is the original gene to cultivate the high-level talents. In-depth studies of classical theories of traditional Chinese medicine such as Inner Canon of Huangdi, Treatise on Febrile Diseases has played an irreplaceable role in inheriting and carrying forward the traditional Chinese medicine characteristics, improving the academic level of TCM talents and the overall quality of the TCM professionals and clinical efficacy ${ }^{[9]}$.

\section{E. The modernization of the studio construction for the}

\section{famous experts in the traditional Chinese medicine}

In the face of the theory of traditional Chinese medicine, it is needed to note in the process of constructing the studios for the famous experts in traditional Chinese medicine that modernization is not westernization, the combination of traditional Chinese medicine and Western medicine is not a simple combination, but the Chinese medicine is the first, western medicine is the second, in the clinical features, it shows the significant efficacy of traditional Chinese medicine treatment. It is needed to set up full-time office secretary and network staff, fully use the modern imaging technology, computer technology and network technology, capture the working process of the normal diagnosis and treatment of the famous experts in traditional Chinese medicine, save the original medical records, input medical information, count up and dig the dialectical thought and drug laws of the famous experts in traditional Chinese medicine in the process of diagnosis and treatment, summarize the clinical experience, and establish a network studio platform, timely upload the cases, medication, lectures, film and television information of traditional Chinese medicine of the famous experts so as to provide domestic and foreign scholars of traditional Chinese medicine and the patients with the sharing information name of the famous experts' application of theory of traditional Chinese medicine to treat disease in traditional Chinese medicine ${ }^{[10]}$. The mentoring students integrate collection, storage, analysis, management as a whole, use the utility software "Being Easy with the Help of Teacher" installed in mobile phones, iPad tablet terminal to achieve information fast acquisition, data storage, quality control management, search inspection and analysis of mining in the clinical experience with the exerts. The clinical experience and academic thought research of the famous experts in traditional Chinese medicine is used as the technical support, which provides the researchers of the inheritance of traditional Chinese medicine with study and application tools, forming the collaboration work platform with full functions for the famous experts in traditional Chinese medicine.

\section{THE PROBLEMS FACED IN THE STUDIO \\ CONSTRUCTION AND ITS SOLUTIONS}

The cultivation modes of the studio of the famous experts in traditional Chinese medicine for the inheritors 
are as follows: the mentoring-type inheritance, family-type inheritance, and graduate education -type inheritance; the former two ones are the typical inheritance ways with Chinese characteristics. However, many too patients, and too much workload of the famous experts make less communication and contact time between experts and students, which causes them cannot carry out full academic and exchanges and experience inheritance; Graduate education exists the disadvantages such as: students pay too much attention to scientific research, ignore clinical experience; the requirements of academic certificate and practitioner qualification in reality cause the limitation for the family-type inheritance development of the traditional Chinese medicine; it is extremely rare for family-type traditional Chinese medicine (TCM) in the real sense, which is unfavorable to the development of TCM. The inheritors of traditional Chinese medicine (TCM) should pay attention to cultivate a firm belief, only they believe in traditional Chinese medicine can they better inherit and develop the traditional Chinese medicine ${ }^{[11]}$. With the rapid development of modern medicine, the students of the Chinese medicine colleges and universities increasingly westernize, they are not familiar with classic of traditional Chinese medicine (TCM), and they lack humanities accomplishment, making it hard for Chinese medicine students to skillfully apply the theory of syndrome differentiation of traditional Chinese medicine to clinical practice. Schools of traditional Chinese medicine not only need to be passed down from one generation to another generation, on the basis of summarizing refining academic ideas, it is needed for them to raise their academic level and clinical practice after the verification. More importantly, it is needed to put forward new ideas on the basis of it, so as to promote the development of traditional Chinese medicine.

\section{CONCLUSIONS}

The development of the inheritance education has played a great role in promoting the study methods of the traditional Chinese medicine, the consolidation and expansion of professional knowledge, the mutual promotion of theory and practice, the construction of the syndrome differentiation and treatment of clinical thinking, the cultivation of personal skills, and the improvement of their own quality, the guidance and cultivation of the inheritors, the learning of the inheritors and increase of the skills levels, which has laid a solid foundation for the inheritors to better adapt to clinical practice, and is a shortcut to be successful in the clinical practice in traditional Chinese medicine (TCM). It is needed to conscientiously sum up the good experience and methods in TCM inheritance education from the beginning, carry out various forms of implementing the specific work, and constantly improve them according to the specific circumstances. The words and deeds of the famous experts in traditional Chinese medicine will promote the development of Chinese medicine talents, and will be passed down to the next generation.

Supported by the national science and Technology Department, and after the Tenth Five-Year Plan rescue inheritance and the 11th Five-Year Plan in-depth study, the clinical experience and academic thinking inheritance research of the famous experts in traditional Chinese medicine not only produced a large number of research results, but also cultivated a large number of outstanding Chinese medicine clinical talents, drove the development of traditional Chinese medicine clinical, promoted the inheritance and innovation of traditional Chinese medicine theory, and had a good social impact, received high evaluation and support from the famous experts in traditional Chinese medicine, researchers and related government departments. During "the Twelfth Five-Year" period, based on the previous study, the inheritance methods and application research of the academic experience of traditional Chinese medicine will be highlighted step by step. The inheritance research on the clinical experience, academic thought of traditional Chinese medicine is a major initiative to promote academic development of TCM and a long-term strategic task. This requires we should constantly explore the inheritance methods and the promotion application methods for the different contents in the process of inheritance research, establish long-term mechanism for research -type inheritance, and promote the Chinese medicine clinical and academic progress.

\section{REFERENCES}

[1] Bai Hua, Xu Chunbo, Gu Xiaojing, Bao Wenhu. The Construction and Implementation of a Comprehensive Information Management Platform for the Inheritance of the Famous Experts in Chinese Traditional Chinese Medicine. . World Chinese Medicine. 2012. 07(2): 96-98

[2] Zhang Boli. Several Opinions on the Academic Thought Organization of the Famous Experts in Chinese Medicine_-Speech at the Forum on Chen Keji's academic thoughts. Chinese Journal of Integrative Medicine. 2011. 31(8): 1013-1015.

[3] Xu Lili, Xue Yanxing, Zhang Runshun, et al. Exploration and Practice of the Research on the Effective Experience of the Famous Experts in Traditional Chinese Medicine. Chinese Journal of Experimental Medicine. 2015. 21(7): 1-4.

[4] Xu Chunbo, Hao Weiwei, Gu Xiaojing, Shao Qian. Research on Inheritance of Clinical Experience and Academic Thought of the Famous Experts in Traditional Chinese Medicine. World Chinese Medicine. 2010. 05(4): 296-298.

[5] Zhu Zhen, Zhang Shiqin, Jin Guilan, Hu Yufeng. To strengthen the ability of traditional Chinese medicine in the traditional Chinese medicine thinking training platform. Laboratory Research and Exploration. 2014. 33(10): 176-179,278.

[6] Wang Yonggang, You Jinzhi, Cui Shulan, Yu Fuli. Individual Characteristics Research Thought of Medical Records of the Famous Experts in Traditional Chinese Medicine. Shaanxi Traditional Chinese Medicine.. 2013. 34(8): 1035-1037.

[7] Yang Guanghua, Wang Ping, Huang Lixing, Liu Yingfeng, Lu Chunzong, Yu Junying. Analysis of the Research Methods of Academic Inheritance of the Famous Experts in Traditional Chinese Medicine. Chinese Journal of traditional Chinese Medicine. 2014. 55(21): 1810-1812.

[8] Chen Xiaofang, Hao Xiaoxiao, Wang Xiaoning, Zhu Fangshi, Chen Jionghua. On the Academic Experience Inheritance of the Famous Experts in Traditional Chinese medicine and the Cultivation of Talents and the Team Construction of Chinese traditional medicine. Chinese Journal of traditional Chinese Medicine. 2013. 54(6): 456-459.

[9] Zhang Yue, Liu Zheng, Cheng Zhixin. Brief Analysis of the Research Status of the Traditional Chinese Medicine Inheritance Methods. Inner Mongolia traditional Chinese Medicine. 2013. 32(31): 142,148.

[10] Li Na. Strengthening the Management of Traditional Chinese Medical Records. Management Observation. 2014. (27): 185-186. 